\title{
CLOSED MAPPINGS OF NOWHERE LOCALLY COMPACT METRIC SPACES
}

\author{
S. A. STRICKLEN, JR.
}

\begin{abstract}
We present a method of construction which allows us to construct many examples of nowhere first countable spaces which are images of metric spaces under closed mappings, and which allows us to prove (with a certain natural restriction on the mapping) that there is a closed mapping from a metric space $M$ onto a space with a dense nowhere first countable subspace if and only if $M$ is nowhere locally compact.
\end{abstract}

1. Introduction. A closed mapping is a continuous function under which the image of a closed set must be closed. In the following, let $M$ be a metric space, $f$ a closed mapping defined on $M$, and write $f(M)=X \cup Y$, where $f(M)$ is first countable at each point of $X$ and at no point of $Y$.

The literature yields two examples in which $f(M)=Y$; the original example of Lašnev [2, example following Theorem 3], and an example of $\mathrm{K}$. R. Van Doren [6, Example 3.3, p.27]. It follows from a theorem of K. Morita [3, Theorem 4(a)] that $Y$ must be closed and discrete if $M$ is locally compact, and Lašnev shows [2, Theorem 2] that $Y$ must be the countable union of closed and discrete subsets of $f(M)$. Van Doren shows [7, Theorem 1] that if $M$ is complete, then $X$ must be dense in $f(M)$ and must (as a metric space) be complete.

We continue this line of research by presenting here a method of construction which allows us to construct many examples in which $f(M)=Y$. We call $f$ irreducible if each open set of $M$ includes some $f^{-1}(p), p \in f(M)$. This is a variation of a definition of Lašnev [2]. Our main result is

THEOREM 1. There exists an irreducible closed mapping $f$ from a metric space $M$ onto a space $f(M)$ with a dense nowhere first countable subspace if and only if $M$ is nowhere locally compact.

2. Upper semicontinuous decompositions. The following variation of $\mathrm{R}$. $\mathrm{L}$. Moore's definition is equivalent to the usual definition in the case of metric spaces:

Definition 1. A collection $H$ of subsets of a metric space $M$ is an upper semicontinuous decomposition (uscd) of $M$ if $\cup H=M$ and if for each $k \in H$,

Presented to the Society, March 21, 1975; received by the editors January 17, 1975 and, in revised form, August 29, 1976.

AMS (MOS) subject classifications (1970). Primary 54B15, 54C10; Secondary 54E35.

Key words and phrases. Nowhere first countable spaces, Lašnev spaces, upper semicontinuous decompositions.

(1) American Mathematical Society 1978 
the following holds: if for each positive integer $l, k_{i} \in H-\{k\}$ and each of $p_{i}$ and $q_{i}$ is a point of $k_{i}$, and if the sequence $p_{1}, p_{2}, \ldots$ converges to a point of $k$, then the set $\left\{q_{1}, q_{2}, \ldots\right\}$ has a limit point in $k$. By considering degenerate sequences, one can show that each element of $H$ is closed and no two elements intersect.

If $H$ is an uscd of the metric space $M$, then the uscd space generated by $H$ is the space in which "point" means "element of $H$ " and in which the topology is generated by a basis consisting of all the sets of the form $\{k \in H \mid k \subseteq D, D$ open in $M\}$.

As is well known, the natural function from $M$ onto the uscd space generated by $H$ is a closed mapping, and for each closed mapping $f$ defined on $M$, the set $H=\left\{f^{-1}(p) \mid p \in f(M)\right\}$ is an uscd of $M$ which generates an uscd space homeomorphic to $f(M)$.

The notable theorem of A. H. Stone [5, Theorem 1] and K. Morita and S. Hanai [4] says that $f(M)$ is metrizable iff $X=f(M)$, and this holds iff for each point $p$ of $f(M), f^{-1}(p)$ has a compact boundary. It is implicit in this theorem that $Y$ is precisely the collection of points $p$ of $f(M)$ such that $f^{-1}(p)$ has a noncompact boundary. Thus the distribution of the points of $Y$ in $f(M)$ is the "same" as the distribution of the elements of the uscd $H=\left\{f^{-1}(p) \mid p\right.$ $\in f(M)\}$ with noncompact boundaries.

3. Construction of upper semicontinuous decompositions. For a closed subset $F$ of a metric space $(M, d)$ we denote by $\operatorname{diam}(F)$ the number $\sup \{d(x, y) \mid x, y \in F\}$; we denote by $S(F ; \varepsilon)$ the set $\{p \in M \mid d(p, q)<$ $\varepsilon$, some $q \in F$ \}. A collection $V$ of subsets of a topological space $T$ is discrete if for each point $p$ of $T$, there is an open set $R$ of $T$ which contains $p$ and intersects at most one element of $V$.

THEOREM 2. If for each positive integer $i, H_{i}$ is a collection of subsets of the metric space $M$ such that:

(1) $\cup H_{i}$ is closed and $H_{i}$ is an uscd of $\cup H_{i}$,

(2) $\cup H_{i}$ does not intersect $\cup H_{j}$ for $i \neq j$, and

(3) if $k \in H_{i}$ then $\operatorname{diam}(k)<1 / i$,

and if we let $H^{\prime}=H_{1} \cup H_{2} \cup \ldots$, then $H=H^{\prime} \cup\left\{\{p\} \mid p \in M-\cup H^{\prime}\right\}$ is an uscd of $M$.

Proof Let $k \in H$, and for each positive integer $i$, let $k_{i} \in H-\{k\}$ and $p_{i}$, $q_{i} \in k_{i}$. Assume $p_{1}, p_{2}, \ldots$ converges to a point of $k$. By properties (1) and (2) of the hypothesis, if for infinitely many positive integers $n, k_{n} \in H_{j}$ for some fixed $j$, then $p \in \cup H_{j}$, so $k \in H_{j}$, and $\left\{q_{1}, q_{2}, \ldots\right\}$ has a limit point in $k$ since $H_{j}$ is an uscd of $\cup H_{j}$. If for each positive integer $n, H_{n}$ contains at most finitely many of $k_{1}, k_{2}, \ldots$, then from hypothesis (3) and the fact that each $k_{i}$ is either in $H^{\prime}$ or is a single point, it follows that $\lim _{n=1,2, \ldots}\left(\operatorname{diam}\left(k_{n}\right)\right)$ $=0$, so that $p$ is a limit point of $\left\{q_{1}, q_{2}, \ldots\right\}$ and Definition 1 is satisfied.

The following corollary is a weaker version of Theorem 2 , and is stated because it is used extensively in the constructions below. 
COROllaRy 2.1. If for each positive integer $i, H_{i}$ is a discrete collection of closed subsets of $M$ such that:

(1) $\cup H_{i}$ does not intersect $\cup H_{j}$ for $i \neq j$, and

(2) for $k \in H_{i}$, $\operatorname{diam}(k)<1 / i$,

and we let $H^{\prime}=H_{1} \cup H_{2} \cup \ldots$, then $H=\left\{\{p\} \mid p \in M-\cup H^{\prime}\right\}$ is an uscd of $M$.

The above results are foreshadowed by the idea of topologically contracting in $[1$, p. 285]. The definition there requires, in effect, that each element of an uscd be compact, however.

Proof of THE ONLY IF OF THeOREM 1. If there is an irreducible closed mapping $f$ from $M$ onto a space $f(M)$ which has a dense, nowhere first countable subspace $T$, then, by definition of irreducible, if $p \in M$ and $R$ is an open set containing $p$, there is a point inverse $f^{-1}(q), q \in f(M)$, included in $R$. Either $q \in T$ or $q$ is a limit point in $f(M)$ of $T$. In either case, $R$ includes a point inverse $f^{-1}(x), x \in T$, which has a noncompact boundary. Thus $M$ is not locally compact at $p$.

Proof OF THE If PART OF TheOREM 1 . We first observe that if $M$ is nowhere locally compact, then $M$ is perfect (i.e. $M$ has no isolated points), since $M$ would be locally compact at any isolated point. For convenience in starting the induction below, we use throughout the bounded distance function $d$ defined by $d(x, y)=\frac{1}{2}$ if $d^{\prime}(x, y)>\frac{1}{2}$ and $d(x, y)=d^{\prime}(x, y)$ otherwise. This does not change the topology on $M$ or on any uscd of $M$.

If $F$ is a subset of $M$, we use the notation $\operatorname{cl}(F)$ for the closure of $F$, and $\operatorname{int}(F)$ for the interior of $F$.

We shall construct by induction a sequence $i(1), i(2), \ldots$ of positive integers, and sequences $A_{1}, A_{2}, \ldots$ and $H_{1}, H_{2}, \ldots$ of collections of subsets of $M$ such that for each positive integer $n$,

(1) $i(n) \geqslant n$,

(2) $H_{n}$ is a collection of infinite closed and discrete point sets such that for any positive integer $j<n$ (if such exist), $\cup H_{n}$ does not intersect $\cup H_{j}$,

(3) $\cup H_{n}$ is closed and discrete,

(4) $A_{n}$ is a maximal collection of closed balls about points of $M$ having radius $1 / i(n)$ such that no two balls of $A_{n}$ intersect and such that $\cup A_{n}$ contains no point of $\cup H_{j}$ for any positive integer $j<n$, and

(5) for each closed ball $s$ of $A_{n}$ there is a point $x_{s}$ such that $s=$ $\operatorname{cl}\left[S\left(x_{s} ; 1 / i(n)\right)\right]$ and such that $\operatorname{cl}\left[S\left(x_{s} ; 1 /(4 \cdot i(n))\right)\right]$ includes exactly one element of $H_{n}$.

We define $i(n)=1$. We define $A_{1}=\{M\}$ and we define a set $k(1, M)$ by selecting a point $x$ of $M$ and letting $k(1, M)$ be an infinite closed and discrete set lying in $\operatorname{cl}\left[S\left(x ; \frac{1}{4}\right)\right]$. There must exist such a $k(1, M)$ because $M$ is not locally compact at $x$. Let $H_{1}=\{k(1, M)\}$. Then, since the bounded distance is used, $i(1), A_{1}$, and $H_{1}$ all satisfy (1)-(5).

Now suppose that $n>1$ is an integer and for each integer $j, 0<j<n$, that $i(j), A_{j}$, and $H_{j}$ have been constructed to satisfy (1)-(5). From (3), 
$\left(\cup H_{1}\right) \cup \cdots \cup\left(\cup H_{n-1}\right)$ is closed and discrete, so there is a point $p \in M$ which is not a limit point of $\left(\cup H_{1}\right) \cup \cdots \cup\left(\cup H_{n-1}\right)$ since $M$ is perfect. We define $i(n)$ to be the least integer larger than $i(n-1)$ such that there is a point $x$ of $M$ such that $\operatorname{cl}[S(x ; 1 / i(n))]$ contains no point of $\left(\cup H_{1}\right)$ $\cup \cdots \cup\left(\cup H_{n-1}\right)$.

By Zorn's Lemma, there is a maximal collection $A_{n}$ of closed balls about points of $M$ with radius $1 / i(n)$ such that no two closed balls of $A_{n}$ intersect and no closed ball of $A_{n}$ contains a point of $\left(\cup H_{1}\right) \cup \cdots \cup\left(\cup H_{n-1}\right)$.

For each closed ball $s=\operatorname{cl}\left[S\left(x_{s} ; 1 / i(n)\right)\right]$ of $A_{n}$, the ball $S\left(x_{s} ; 1 /(4 \cdot i(n))\right)$ contains an infinite closed and discrete set $k(n, s)$, since $M$ is not locally compact at $x_{s}$. We let $H_{n}=\left\{k(n, s) \mid s \in A_{n}\right\}$.

From the construction it is clear that $i(n), A_{n}$, and $H_{n}$ satisfy (1), (4), and (5) above. If $p \in \operatorname{int}(s)$, some $s \in A_{n}$, then $p$ is not a limit point of $k(n, s)$, nor is $p$ a limit point of the set $\cup\left\{k \in H_{n} \mid k \notin s\right\}$. If $p \notin \operatorname{int}(s)$ for any $s \in A_{n}$, then $S(p ; 1 /(4 \cdot i(n)))$ is an open set containing $p$ and intersecting none of the elements of $H_{n}$. In either case, $p$ is not a limit point of $\cup H_{n}$ so (2) and (3) are satisfied. This concludes the induction.

We define $H^{\prime}=H_{1} \cup H_{2} \cup \ldots$ and we define $H=H^{\prime} \cup\{\{p\} \mid p \in M$ $\left.-\cup H^{\prime}\right\} . H$ fits the hypotheses of Theorem 2, so $H$ is an uscd of $M$. No element of $H^{\prime}$ has a compact boundary so the uscd space generated by $H$ is not first countable at any of the points corresponding to elements of $H^{\prime}$. It remains to be shown that the natural mapping from $M$ onto the uscd space generated by $H$ is irreducible, and that the image of $H^{\prime}$ under this mapping is dense in the uscd space. Both of these will be shown if we show that for each $p \in M$, every ball containing $p$ covers an element of $H^{\prime}$.

Let $p \in M$ and let $s$ be a ball containing $p$. There is an integer $t$ such that $S(p ; 1 / t) \subseteq s$. Assume that no element of $H^{\prime}$ is included in $s$. Then no closed ball of any $A_{n}$ for $n>2 t$ intersects $S(p ; 1 / 2 t)$, since such a ball would, by (1) have a radius less than $1 / 2 t$ and so lie in $S(p ; 1 / t)$ implying from (5) that $s$ includes an element of $H_{n}$.

Thus the only elements of $H^{\prime}$ intersecting $S(p ; 1 / 2 t)$ are in $H_{1} \cup H_{2}$ $\cup \cdots \cup H_{2 t}$. The union of this collection is closed and discrete by (3), and $M$ is perfect, so there is a point $q$ in $S(p ; 1 / 2 t)$ which is not in $\cup H^{\prime}$ and there is an integer $j>2 t$ such that $S(q ; 1 / j)$ lies in $S(p ; 1 / 2 t)$ and intersects no element of $A_{j}$. This contradicts the maximality of $A_{j}$ guaranteed in (4). This concludes the proof. Note that the construction of $S(q ; 1 / j)$, in particular the italicized part, depends heavily on metrizability, so that this approach is unlikely to work for more general spaces.

The statement that $f$ is irreducible is equivalent to the statement that for no proper closed subset $K$ of $M$ is $f \mid K(K)=f(M)$. It follows from a theorem of Lašnev [4, Theorem 4] that there is always a (not necessarily proper) closed subset $K$ of $M$ such that $f \mid K(K)=f(M)$, so that if we require $f$ to be irreducible, we still consider all images of metric spaces under closed mappings. The following example shows the importance of the irreducibility hypothesis in Theorem 1 . 
EXAMPLE 1. Let $A_{1}$ be a collection of closed, nonintersecting circular discs in the plane, each having radius 1 , and let $A_{1}$ be maximal in the sense that any closed circular disc of radius 1 in the plane intersects an element of $A_{1}$. Remove from the boundary of each element of $A_{1}$ all but a countable dense subset. This collection of subsets of closed discs is $H_{1}$. If $H_{n}$ is defined, let $A_{n+1}$ be a collection of closed, nonintersecting circular discs in the plane each of radius $\frac{1}{10} \cdot n$, no one of which intersects any element of any $A_{j}$ for $0<j \leqslant n$, and let $A_{n+1}$ be maximal in the sense that any closed circular disc in the plane having radius $\frac{1}{10} \cdot n$ intersects some element of some $A_{j}, 0<j \leqslant$ $n+1$. Remove from the boundary of each element of $A_{n+1}$ all but a countable dense collection of points. This collection of subsets of closed circular discs is $H_{n+1}$. Define $M=\cup\left\{\cup H_{i} \mid i=1,2, \ldots\right\}$ under the relative topology induced by the plane. Define $H=\cup\left\{H_{i} \mid i=1,2, \ldots\right\}$. The hypotheses of Corollary 2.1 are satisfied, and each element of the uscd has a noncompact boundary, so the uscd space is nowhere first countable. Except for the countable complement of a dense open set, $M$ is locally compact, and is a two-manifold.

EXAMPLE 2. $M$ is the subspace of the real plane consisting of those points $(a, b)$ in the interior of the unit square such that $a$ is a diadic rational and $b$ is not a diadic rational which (when both are expressed in lowest terms) has a denominator less than or equal to that of $a$. The elements of the decomposition $H$ are the components of $M$. The hypotheses of 2.1 are satisfied. Each element of the uscd has a noncompact boundary, so the uscd space is nowhere first countable. The natural mapping is irreducible in this case, and each point inverse is connected.

Lašnev's example cited above consists of the subspace $M^{\prime}$ of all points $(a, b)$ in $M$ such that $b$ is irrational. The decomposition $H^{\prime}$ of $M^{\prime}$ is defined by $H^{\prime}=\left\{k \cap M^{\prime} \mid k \in H\right\}$. In this case, each point inverse is totally disconnected but is metrically complete.

EXAmple 3. Let $M$ be the space of rational numbers. Denumerate the points in $M$ by $r_{1}, r_{2}, \ldots$. Place a segment of length no greater than 1 about $r_{1}$, and select a closed (in $M$ ) and discrete sequence $r_{1}^{1}, r_{2}^{1}, \ldots$ of points in that segment. Let $k_{1}=\left\{r_{1}, r_{1}^{1}, r_{2}^{1}, \ldots\right\}$ and let $H_{1}=\left\{k_{1}\right\}$.

If $H_{n}$ and $k_{n}$ have been defined, let $r_{1}^{n+1}$ be the first element of $r_{1}, r_{2}, \ldots$ not in $k_{1} \cup \cdots \cup k_{n}$. Since $k_{1} \cup \cdots \cup k_{n}$ is closed and discrete, there is a segment of length no greater than $1 /(n+1)$ containing $r_{1}^{n+1}$ and no point of $k_{1} \cup \cdots \cup k_{n}$. Select a closed and discrete sequence $r_{2}^{n+1}, r_{3}^{n+1}, \ldots$ from that segment. Let $k_{n+1}=\left\{r_{1}^{n+1}, r_{2}^{n+1}, \ldots\right\}$ and let $H_{n+1}=\left\{k_{n+1}\right\}$. The hypotheses of 2.1 are satisfied and $H=H_{1} \cup H_{2} \cup \ldots$ exhausts $M$. Each element of $H$ has a noncompact boundary, so the natural mapping is a closed continuous function from the space of rationals onto a nowhere first countable space. In this case, each point inverse is closed and discrete.

It seems clear that many examples of closed mappings of metric spaces onto nowhere first countable spaces can be constructed in this fashion. 


\section{REFERENCES}

1. R. L. Moore, Foundations of point set theory, rev. ed., Amer. Math. Soc. Colloq. Publ., vol. 13, Amer. Math. Soc., Providence, R. I., 1962. MR 27 \# 709.

2. N. Lašnev, Continuous decompositions and closed mappings of metric spaces, Dokl. Akad. Nauk SSSR 165 (1965), 756-758 = Soviet Math. Dokl. 6 (1965), 1504-1506. MR 33 \# 703.

3. K. Morita, On closed mappings, Proc. Japan Acad. 32 (1956), 539-543. MR 19, 49.

4. K. Morita and S. Hanai, Closed mappings and metric spaces, Proc. Japan Acad. 32 (1956), 10-14. MR 19, 299.

5. A. H. Stone, Metrizability of decomposition spaces, Proc. Amer. Math. Soc. 7 (1956), 690-700. MR 19, 299.

6. K. R. Van Doren, Concerning closed, continuous images of metric spaces, Ph.D. Thesis, Auburn Univ., Auburn, Ala., 1972.

7. $\ldots$ Closed, continuous images of complete metric spaces, Fund. Math. 80 (1973), 47-50. MR 50 \# 11133.

Department of Mathematics, Southern Technical Institute, Marietta, Georgia 30060 